\section{A FORTRAN program for statistical evaluation of pseudorandom number generators}

\author{
JEFFREY LEE RASMUSSEN \\ University of Arizona, Tucson, Arizona
}

There are numerous applications in the field of psychology that require the use of random numbers. Randomization of subjects into groups, random presentation of treatments to subjects, simulation of cognitive processes, and Monte Carlo simulations of statistics used in psychological research require the use of random numbers. A common procedure for obtaining random numbers is through the use of a computer's pseudorandom number function. The function is deemed pseudorandom because the values are not generated from a truly random process; rather, each value is calculated from the previous value, often through multiplicative and modulo operations. In order to be useful, the values produced by a pseudorandom number generator must meet certain criteria of randomness. Computer companies usually do not supply users with information concerning the adequacy of their pseudorandom number generator in meeting these criteria. It is well known that some pseudorandom number generators do not produce satisfactory random numbers (Jansson, 1966; Knuth, 1969). It is therefore the user's responsibility to determine the adequacy of a pseudorandom number generator.

The present article describes a FORTRAN IV program that may be used to test the adequacy of a pseudorandom number generator. It checks whether the values produced by the generator pass a cycle repetition test plus four standard statistical tests: rectangularity test, sequential independence test, runs up and down test, and gaps test. Each test is conducted on the same 100,000 random values. The program output consists of a probability value for each of the statistical tests. The tests are described in numerous sources (Hammersley \& Handscomb, 1964; Jansson, 1966; Knuth, 1969; Lehman, 1977; Shreider, 1966). Of these references, Jansson (1966) and Lehman (1977) present the tests in the clearest fashion.

Cycle Repetition. Due to the manner in which pseudorandom values are generated, most pseudorandom number generators produce a finite series of different pseudorandom numbers. At the end of a certain cycle, the generator will reproduce the same sequence of numbers. For a pseudorandom number generator to be useful, it must generate an acceptably long series of numbers before it repeats its cycle. What constitutes an "acceptably long series" depends upon

The author's mailing address is: Department of Psychology, University of Arizona, Tucson, AZ 85721. the particular application for which the numbers are to be used. For example, a cycle length of 200 is suitable for randomly assigning 200 subjects to treatment conditions, but is not suitable for a Monte Carlo simulation involving tens of thousands of numbers. The present program warns the user if the pseudorandom number generator repeats its cycle before 100,000 repetitions.

Rectangularity Test. The rectangularity test, also referred to as the frequency test, checks whether the generator's output departs from a uniform distribution. The test is carried out by dividing the range of the possible values into 10 equal intervals. The program counts the number of values that fall in each interval. A chi-square goodness-of-fit test is computed and converted to its probability value by a routine by Dunlap and Duffy (1975). A low probability value (e.g., $<.05$ or $<.01$ ) indicates that the pseudorandom number generator produces numbers that have a distribution that deviates significantly from a uniform distribution.

Sequential Independence Test. This test checks whether a correlation exists between values that share the same lagged relationship in a sequence of numbers. For example, the sequence $1,4,9,1,5,8,2,4,8,1,5,9$ shows a strong correlation $(r=.95)$ between values of $x_{i}$ and $x_{i+3}$. The sequential independence test is carried out by calculating the average lagged product for lags of length $k$, from $k=1$ (adjacent pairs) to $k=50$ on a sequence of $\mathrm{N}-\mathrm{k}$ numbers. The program outputs the probability value for each of the 50 lags. A small probability value for a given lag indicates that the generator does not show sequential independence for that lag.

Runs Up and Down Test. The runs test checks whether the generator produces values that tend to increase or decrease in a random fashion. For the runs test, the program counts the number of runs of length $k$, where a run is defined as a series of values where $x_{i}<$ $x_{i+1}$ (ascending run) or $x_{i}>x_{i+1}$ (descending run) for $k$ consecutive values. For example, the sequence $1,6,8,9,2,4,7$ shows an ascending run of length 3 , followed by a descending run of length 1 , followed by an ascending run of length 2 . (Lehman, 1977, p. 139, incorrectly denotes the sequence $.15, .23, .65, .66$ as an example of a run of length 4 . This should be denoted as a run of length 3-cf. Levene \& Wolfowitz, 1944.) A chi-square goodness-of-fit test is calculated and converted to its probability value. A low probability value for the runs test indicates that the values generated do not increase or decrease in a random fashion.

Gaps Test. The gaps test checks whether values from a pseudorandom number generator are too closely packed or too spread out. In order to conduct the gaps test, the program first converts the real random values produced by the generator to integer values between 1 and 10 inclusive. A gap is defined as the number of values intervening between two occurrences of the same digit. 
For example, in the sequence $6,4,9,6$, there is a gap of two between the two occurrences of the digit 6 . A probability value from a goodness-of-fit test can be used as a measure of how much the obtained number of gaps deviates from the expected number of gaps. A small probability value for the gaps test indicates that the generator produces digits that are packed too closely together or are spread too far apart.

Program Requirements. The program is written in standard FORTRAN IV and was tested on a Control Data Corporation Cyber 175 computer. Two changes may be necessary to make the program run on another computer system. First, on the Cyber the pseudorandom number function is called by RANF(). The name of the function and the number of arguments may differ on other computing systems. Second, on the Cyber the terminal is defined as unit 5 in an OPEN statement; terminal definition will probably be different on other systems. The program takes approximately $7 \mathrm{sec}$ to run on the Cyber.

Program Availability. A listing of the FORTRAN program is available free of charge from Jeffrey Lee Rasmussen, Department of Psychology, University of Arizona, Tucson, AZ 85721.

\section{REFERENCES}

Dunlap, W. P., \& DuFfy, J. A. (1975). FORTRAN IV functions for calculating exact probabilities associated with $\mathrm{z}$, chi-square, t, and F values. Behavior Research Methods \& Instrumentation, 7, 59-60.

Hammersley, J. M., \& Handscomb, D. C. (1964). Monte Carlo methods. New York: Wiley.

Jansson, B. (1966). Random number generators. Stockholm: Victor Pettersons Bokindustri Aktiebolag.

KNUTH, D. E. (1969). Semi-numerical algorithms. Reading, MA: Addison-Wesley.

Lehman, R. S. (1977). Computer simulation and modeling: An introduction. Hillsdale, NJ: Erlbaum.

LeVENe, H., \& Wolfowitz, J. (1944). The covariance matrix of runs up and down. Annals of Mathematical Statistics, 15, 58-69.

Shreider, Y. A. (1966). The Monte Carlo method. Oxford: Pergamon Press.

(Revision accepted for publication January 30, 1984.) 